\title{
Monitoring Fetal Heart Rate during Pregnancy: Contributions from Advanced Signal Processing and Wearable Technology
}

\author{
Maria G. Signorini, ${ }^{1}$ Andrea Fanelli, ${ }^{2}$ and Giovanni Magenes ${ }^{3}$ \\ ${ }^{1}$ Dipartimento di Elettronica, Informazione e Bioingegneria (DEIB), Politecnico di Milano, piazza Leonardo da Vinci 32, \\ 20133 Milano, Italy \\ ${ }^{2}$ Computational Physiological and Clinical Inference Group, 143 3rd Street, Apt. 1, Cambridge, MA 02141, USA \\ ${ }^{3}$ Dipartimento di Ingegneria Industriale e dell'Informazione, University of Pavia, Via A. Ferrata 1, 27100 Pavia, Italy
}

Correspondence should be addressed to Maria G. Signorini; mariagabriella.signorini@polimi.it

Received 15 July 2013; Revised 29 October 2013; Accepted 10 November 2013; Published 30 January 2014

Academic Editor: Mihaela Ungureanu

\begin{abstract}
Copyright ( 2014 Maria G. Signorini et al. This is an open access article distributed under the Creative Commons Attribution License, which permits unrestricted use, distribution, and reproduction in any medium, provided the original work is properly cited.

Monitoring procedures are the basis to evaluate the clinical state of patients and to assess changes in their conditions, thus providing necessary interventions in time. Both these two objectives can be achieved by integrating technological development with methodological tools, thus allowing accurate classification and extraction of useful diagnostic information. The paper is focused on monitoring procedures applied to fetal heart rate variability (FHRV) signals, collected during pregnancy, in order to assess fetal well-being. The use of linear time and frequency techniques as well as the computation of non linear indices can contribute to enhancing the diagnostic power and reliability of fetal monitoring. The paper shows how advanced signal processing approaches can contribute to developing new diagnostic and classification indices. Their usefulness is evaluated by comparing two selected populations: normal fetuses and intra uterine growth restricted (IUGR) fetuses. Results show that the computation of different indices on FHRV signals, either linear and nonlinear, gives helpful indications to describe pathophysiological mechanisms involved in the cardiovascular and neural system controlling the fetal heart. As a further contribution, the paper briefly describes how the introduction of wearable systems for fetal ECG recording could provide new technological solutions improving the quality and usability of prenatal monitoring.
\end{abstract}

\section{Introduction}

Monitoring biomedical signals, through measurement, quantification, evaluation, and classification of signal properties, is one of the primary tools for investigating the evolution of disease states. The overall architecture of a monitoring system has to combine technological tools with signal analysis methods in order to extract useful information to identify patient's condition.

Inside these procedures, it is very important to select processing methods that can enhance pathophysiological signal properties, thus linking parameters to physiological events (and maybe to physical quantities).

Traditional monitoring systems received a fundamental improvement by new technological devices allowing longer and deeper data collection as well as by advanced clinical tools for data interpretation.

In recent years, the development of dynamical system analysis has led to the introduction of a large amount of signal processing techniques aimed at the extraction of parameters from experimental time series, thus enhancing new information about the characteristics of the system generating the time series. In most cases, however, an accurate model of the generating system is unknown or too complex and the output signal is the main available information about the system itself.

A typical example is the cardiovascular system, where the main way to investigate heart function consists of the analysis of heart rate variability signal (HRV). It has been shown that HRV signal can be related to the activity of several 
physiological control mechanisms of different nature. Their interaction produces changes in the beat rate assuring the system controlling heartbeats reacts efficiently to different incoming stimuli. HRV variance is related to changed conditions of heart activity. Frequency domain analysis of the HRV signal provides quantitative and noninvasive measures of the activity of the autonomic nervous system (ANS) [1]. A linear modeling approach is adopted to quantify both the sympathetic and parasympathetic control mechanisms and their balance through the measure of spectral low and high frequency components (LF and HF). The same approach can extract parameters related to the heart and to the cardiovascular control even from systolic and diastolic values in arterial blood pressure (ABP), on a beat-to-beat basis [2].

Nevertheless, even if the HRV analysis through classical linear methods provides the quantification the ANS regulating action in the short period [1], the linear approach cannot explain the whole information carried by beat-tobeat variability [3]. Results on HRV signal analysis show that its dynamic behavior also involves nonlinear components that contribute to the signal generation and control [3, 4]. Signal structure appears erratic but it presents abrupt changes and patterns in which a more regular behavior appears. To investigate the erratic components of the cardiac rhythms and to assess nonlinear deterministic phenomena affecting HRV signal, both in short and long temporal windows, nonlinear signal analysis has demonstrated its usefulness [5].

In the field of fetal heart rate monitoring during pregnancy, linear time and frequency techniques were traditionally adopted. Fetal HR monitoring is a challenging procedure for people working in the obstetric field, in order to check if the fetus is and remains in a wellbeing state as the pregnancy develops.

The most employed diagnostic examination in the clinical practice is cardiotocography (CTG). CTG combines fetal heart rate (FHR) measurement, obtained by means of a Doppler ultrasound probe and uterine contraction, recorded through an abdominal pressure transducer. During pregnancy, each woman undergoes one or more ambulatory monitoring tests and, in the last pregnancy trimester and/or in case of suspect that risky condition can take place, monitoring frequency can increase to weekly or even daily. We can certainly state that the total CTG recording amount, in our country, is about 1 million per year and reaches several million exams in EU countries.

CTG is universally accepted in the clinical practice and it is recognized as one of the most information rich among noninvasive diagnostic tests for prenatal monitoring. Nevertheless, the FHR signal is usually analysed by detecting and measuring morphological characteristics whose clinical relevance is established mainly by eye inspection. This represents a strong limitation because the application of subjective and qualitative methods lacks reliability and depends on the physician experience.

Moreover, the CTG exam needs a hospital context to be performed both as an expert clinician only can produce the clinical report and the technology the system requires for signal recording.
One can state with some confidence that the techniques used in the prenatal diagnosis for FHR analysis did not experience a growth rate as the knowledge did, concerning physiological mechanisms and the availability of methodological tools with clearly demonstrated investigation abilities.

The introduction of quantitative evaluation of both linear and nonlinear indices increases the diagnostic power and reliability of antepartum monitoring.

The paper presents results obtained by applying both linear and nonlinear quantitative analysis to fetal heart rate (FHR) signals collected in normal and intrauterine growth restricted (IUGR) fetuses $(61+61$ subjects).

Finally, as a further contribution, the paper briefly describes the simultaneous development of a new wearable monitoring system allowing comfortable collection of fetal ECG and HRV signals in long periods. This new device named Telefetalcare is equipped with the analysis tools developed for the fetal HR analysis and described in this paper, and can provide further improvements to pre-natal diagnostic system tools.

\section{Materials and Methods}

2.1. FHRV Recording. FHRV recordings were collected at the Azienda Ospedaliera Universitaria Federico II, Napoli, Italy. Signals were recorded by means of a Hewlett Packard CTG fetal monitor, linked with a PC computer through a USB port.

The HP fetal monitors use an autocorrelation technique to compare the demodulated Doppler signal of a heartbeat with the next one. Each Doppler signal is sampled at $200 \mathrm{~Hz}$ (5 ms). The time window over which the autocorrelation function is computed is $1.2 \mathrm{sec}$, corresponding to a FHR lower bound of $50 \mathrm{bpm}$. A peak detection software then determines the heart period (the equivalent of RR period) from the autocorrelation function. With a peak position interpolation algorithm, the effective resolution is better than $2 \mathrm{~ms}$.

Due to historical reasons, almost all commercially available fetal CTG monitors display only the fetal heart rate expressed in number of beats per minute (bpm) and do not offer the series of interbeat intervals, usually employed in HRV analysis.

The HP monitor produces a FHR value in bpm every $250 \mathrm{msec}$. In the commercially available system, the PC reads 10 consecutive values from the monitor every $2.5 \mathrm{sec}$ and determines the actual FHR as the average of the 10 values (corresponding to an equivalent sampling frequency of $0.4 \mathrm{~Hz}$ ). We modified the software in order to read the FHR at $2 \mathrm{~Hz}$ (every $0.5 \mathrm{sec}$ ). The choice of reading the FHR values each $0.5 \mathrm{sec}$ represents a reasonable compromise to achieve an enough large bandwidth (Nyquist Frequency $1 \mathrm{~Hz}$ ) and an acceptable accuracy of the FHR signal. An example of CTG recording is shown in Figure 1, where both the FHR and the uterine contractions are plotted as functions of time.

The whole set of recordings was composed of 122 subjects (61 healthy and 61 IUGR). Both groups were defined "a posteriori," after delivery, on the basis of standard parameters (Apgar scores, weight, abdominal circumference): IUGR 


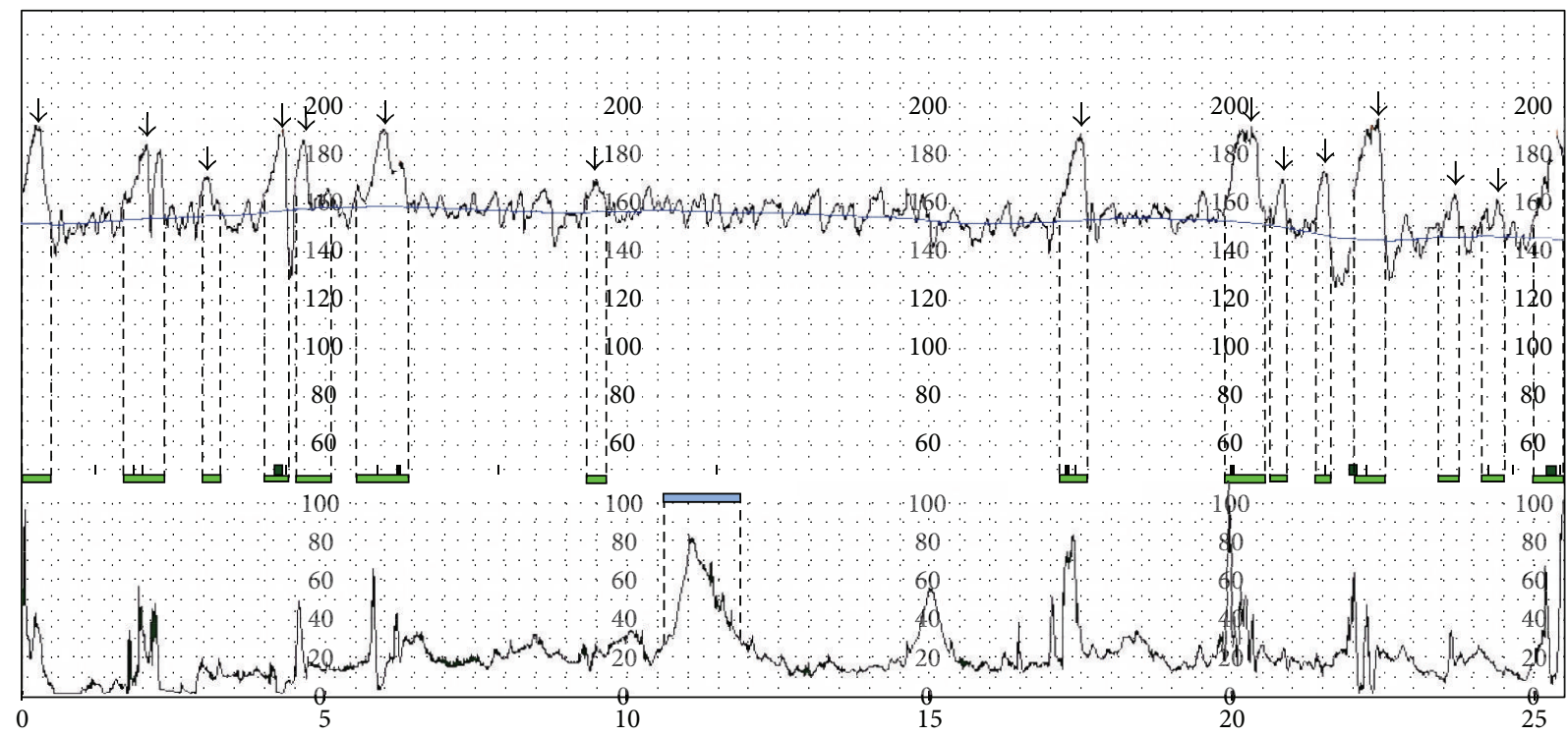

FIGURE 1: Example of CTG graph. The upper trace is fetal heart rate signal obtained by a Doppler ultrasound probe; the baseline is drawn and the arrows represent the detected accelerations. The lower tracing is the toco signal (uterine contractions). Time units are in minutes.

TABLE 1: Detailed summary of the two groups of fetuses.

\begin{tabular}{lcc}
\hline Population details & Healthy & IUGR \\
\hline $\begin{array}{l}\text { Number } \\
\text { Mother age (years) }\end{array}$ & $32.34 \pm 5.64$ & 61 \\
$\begin{array}{l}\text { Gestational age at CTG } \\
\text { recording (days) }\end{array}$ & $34.78 \pm 0.53$ & $32.27 \pm 2.79$ \\
$\begin{array}{l}\text { Gestation age at delivery } \\
\text { (days) }\end{array}$ & $39.74 \pm 1.15$ & $34.15 \pm 2.99$ \\
$\begin{array}{l}\text { Weight of the baby after } \\
\text { delivery }\end{array}$ & $3275 \mathrm{~g} \pm 518 \mathrm{~g}$ & $1479 \mathrm{~g} \pm 608 \mathrm{~g}$ \\
$\begin{array}{l}\text { Delivery mode } \\
58 \% \text { spontaneous } \\
42 \% \text { caesarean }\end{array}$ & $\begin{array}{c}14.8 \% \text { spontaneous } \\
85.2 \% \text { caesarean }\end{array}$ \\
\hline
\end{tabular}

fetuses were selected by weight below the 10th percentile for their gestational age and abdominal circumference below the 10th percentile.

Table 1 summarizes population details. All recordings were made in a controlled clinical environment, with the pregnant woman lying on a bed. The average length of the recordings was $2450 \pm 724 \mathrm{sec}$ for healthy and $3418 \pm 1033 \mathrm{sec}$ for IUGR group.

\subsection{Time and Frequency Domain FHR Analysis}

2.2.1. Baseline, Accelerations, and Decelerations. Interpretation of the heart rate pattern is usually performed by the physician who analyses the deviations of the signal from an imaginary line, the baseline. He/she hypothetically constructs it as a running average of the heart rate. Accelerations and decelerations are defined as deviations from the baseline, and more than one quantitative definition is available. In the construction of an automated system for the evaluation of the CTG recordings, a reproducible determination of the baseline is a fundamental starting point. Several attempts in this direction have been made starting from the work of Dawes et al. [6]; the approach we followed was that suggested by Mantel et al. [7] (an example of baseline is shown in Figure 1). The algorithm is very complex, and a full description can be found in the cited reference.

Accelerations and decelerations are deviations of the fetal heart rate from the baseline lasting a sufficient amount of time (accelerations are positive deviations, decelerations negative). They are correlated with the normal activities of the fetus, who "trains," moves, and exercises to breathe. Decelerations are usually correlated with uterine contraction. Unfortunately, different quantifications of the words "deviations" and "sufficient" led each medical school to develop its own method to evaluate, by means of a ruler, these quantities on the monitoring strip. We applied a quantitative procedure not only fully consistent with the definition of Mantel et al. [8], but also holding the suggestions of Arduini et al. [9].

Classical FHR linear indices are truly time domain measures. In the following, interbeat sequences $T(i), i=$ $1, \ldots, N$, will be used instead of heart rate sequences $S(i)$ in beats per minute, usually employed in cardiotocography: they are computed as $T(i)=60000 / S(i)$ ms. Moreover, in order to be compatible with previous works (Arduini et al. [9]) we also computed some indices on the basis of the undersampled time series $T_{24}(i)=60000 / S_{24}(i) \mathrm{ms}, i=1, \ldots, N / 5$ obtained by taking $S_{24}(i)$ as the average of five consecutive FHR values of $S(i)$.

2.2.2. Long Term Irregularity. Long Term irregularity (LTI) was the first index ever introduced; it was proposed by De Haan et al. [10]. It is usually computed on a three-minute segment of interbeat sequence in milliseconds. We excluded from the computation large accelerations and decelerations, as suggested by Arduini et al. [9], to avoid deviations caused 
by spurious measures of variability. The three minutes, after the removal of the undesired parts, must contain, at least, a continuous segment of 30 seconds.

Given a signal $T_{24}(i)$ with $i \in[a ; b]$, LTI is defined as the interquartile range $[1 / 4 ; 3 / 4]$ of the distribution $m_{24}(j)$ with $j \in[a ; b-1]$ and $m_{24}(j)=\sqrt{T_{24}{ }^{2}(j)+T_{24}{ }^{2}(j+1)}$.

2.2.3. Short Term Variability. Short term variability (STV) quantifies FHR variability over a very short time scale, usually on a beat-to-beat basis. We refer to the definitions provided by Dalton et al. [11] (even if we used a scale factor of 12) and by Arduini et al. [9]. By considering one minute of interbeat sequence, $T_{24}(i)$ in $\mathrm{ms}, i=1, \ldots, 24$, we defined STV as

$$
\begin{aligned}
\mathrm{STV} & =\operatorname{mean}\left[\left|T_{24}(i+1)-T_{24}(i)\right|\right]_{i} \\
& =\frac{\sum_{i=1}^{23}\left|T_{24}(i+1)-T_{24}(i)\right|}{23},
\end{aligned}
$$

where $T_{24}(i)$ is the value of the signal $T(i)$ taken each $2.5 \mathrm{sec}$ (i.e., once each five samples).

2.2.4. Interval Index. Historically, Interval Index (II) was introduced just after LTI and it is certainly one of the most used variability indices. It was proposed by Yeh et al. [12] as a long term variability statistic; we adopted the formulation used by Arduini et al. [9],

$$
\mathrm{II}=\frac{\operatorname{std}\left[T_{24}(i+1)-T_{24}(i)\right]}{\mathrm{STV}}, \quad i=1, \ldots, 23 .
$$

2.2.5. Power Spectral Analysis of Fetal HRV. Considering the FHRV signal as controlled by the ANS, as it happens in adult subjects, it could be of primary importance to own a tool quantifying its development during pregnancy. Literature reports several examples on this subject. The ANS is still developing, if not as the anatomic growth as in the regulatory activity which increases in time with the system maturation.

Estimation of the power spectral density (PSD) in the FHR signal provides parameters related to the ANS activity. Frequency domain FHR analysis adopt both the direct estimation of the periodogram and the autoregressive power spectrum estimation.

In fetal HR analysis it is customary to consider three frequency bands, Low Frequency (LF), Movement Frequency (MF), and High Frequency (HF) power components as well as the ratio $\mathrm{LF} /(\mathrm{MF}+\mathrm{HF})[13]$, instead of the bands usually adopted for standard HRV analysis [1].

Low Frequency contributions (LF: $0.03-0.15 \mathrm{~Hz}$ ) can be associated with the sympathetic control and vasomotor activity. HF is basically driven by respiration mediated by vagal activity (HF: $0.5-1 \mathrm{~Hz}$ ). A third component needs to be considered: we called it Movement Frequency (MF: 0.15$0.5 \mathrm{~Hz}$ ). MF should quantify the activity of the fetus and the mechanical influences of the maternal breathing.

This approach works well on a short time scale (3-5 min, 300 points about) as the stationarity of the fetal HRV signal is an essential requirement. We adopted the autoregressive power spectrum estimation method as described in Signorini et al. [13].

$\mathrm{LF} / \mathrm{HF}+\mathrm{MF}$ ratio could represent a synthetic index of the balance between physiological control components and fetus activity level, representing the equivalent of the socalled sympathovagal balance in standard HRV analysis.

2.3. Nonstandard Parameters for FHR Analysis. The introduction of nonlinear approaches to signal processing led to considering a set of methods investigating geometric and dynamic properties of time series.

Differently from the approach usually adopted to study a well-known deterministic system, when we deal with complex nonlinear systems, very often we can only analyze experimental time series. Nevertheless important indications can be extracted from the parameters estimating nonlinear characteristics. Their statistical use can be of great importance, even in diagnostic field and in clinical knowledge related to different cardiovascular pathologies [5].

Various techniques exist aimed at quantifying the degree of similarity and/or complexity in time series which can be computed directly on the sequence of interbeat intervals [14, $15]$.

2.3.1. Regularity Properties: Entropy Estimators (ApEn, Sam$p E n)$. ApEn index quantifies regularity and complexity of a time series. The index was proposed in [16] and further improvements and corrections were proposed by the introduction of the SampEn index.

The idea is to quantify the degree of regularity or loss of regularity in a time series without a priori information on its structure. ApEn works on short ( $<100$ samples) and noisy time series.

ApEn estimator depends on a parameter $m$ (length of runs compared in the time series) and on a parameter $r$ (percentage of signal std., working as a filter). The $\operatorname{ApEn}(m, r, N)$ evaluates, within a tolerance $r$, the signal regularity, by assessing the frequency of patterns similar to a given pattern of window length $m(m=1,2, r: 0.1-0.25$ std of the input data [16]).

Once values of the two parameters $m$ and $r$ are fixed and given $N$ data points, the procedure constructs sequences $x_{m}(i)$ and computes, for each $i \leq N-m+1$,

$$
\begin{aligned}
C_{i}^{m}(r)=(N-m+1)^{-1}\{ & \text { number of } j \leq N-m+1 \\
& \text { such that } \left.d\left[x_{m}(i), x_{m}(j)\right] \leq r\right\} .
\end{aligned}
$$

Regularity parameter is defined as

$\operatorname{ApEn}(m, r, N)=\lim _{N \rightarrow \infty}\left[\Phi_{m}-\Phi_{m}+1\right]$, where $\Phi^{m}(r)=$ $(N-m+1)^{-1} \sum_{i=1}^{N-m+1} \ln C_{i}^{m}(r)$.

The estimator of this parameter for an experimental time series of a fixed length $N$ is given by $\operatorname{ApEn}(m, r, N)=\left[\Phi_{m}-\right.$ $\left.\Phi_{m}+1\right]$.

Other methods estimate entropy-like indexes in time series. Among them, Sample Entropy (SampEn) has been largely employed in biomedical signal processing over time, as it improves the estimation performed by ApEn (i.e., 
removes the bias introduced by self-counts). SampEn is also the basis for a multiscale approach: entropy parameters are calculated at different scales in coarse-grained time series $[17,18]$.

ApEn and SampEn were estimated in the same time series by using the same parameter set: $m=1$ and $r=0.1, m=2$ and $r=0.15$ and 0.2 .

2.3.2. Lempel Ziv Complexity. Lempel Ziv complexity (LZC) was originally proposed in the information field to assess the complexity of data series [19]. Its measure is associated with the number of different substrings and to the rate of their recurrence. Namely, LZC reflects the gradual increase of new patterns along the given sequence. The measure of complexity introduced by Lempel and Ziv assesses the socalled algorithmic complexity, which is defined according to Information Theory as the minimum quantity of information needed to define a binary string. In case of random strings, the algorithmic complexity is the length of the string itself. In fact any compression effort will produce an information loss. In order to estimate the LZC in a time series, it is necessary to transform the signal (the FHR in or case) into symbolic sequences.

Calculation of the Lempel Ziv complexity $c(n)$ needs to define an alphabet $\mathrm{A}$, that is, the set of symbols which compose the sequence $S$ (for a binary string, A is simply $\{0,1\})$.

Suppose the number of symbols in the alphabet A is $\alpha$ and the length of sequence is $l(S)=n$. The upper bound of $c(n)$ is given by:

$$
c(n)<\frac{n}{\left(1-\varepsilon_{n}\right) \log (n)},
$$

where $\varepsilon_{n}=2(1+\log \log (\alpha n)) / \log (n)$ [6]. When $n$ is large enough $(n \rightarrow \infty), \varepsilon_{n} \rightarrow 0$ and we have that

$$
\lim _{n \rightarrow \infty} c(n)=b(n)=\frac{n}{\log _{\alpha}(n)} .
$$

The quantity $b(n)$ is the asymptotic behaviour of $c(n)$ for a random string. The normalized complexity is thus defined as $C(n)=c(n) / b(n)$.

In order to estimate the complexity measure for the HRV time series, we have transformed the signals in symbolic sequences. As a coding procedure we adopted both a binary and a ternary code. From an HRV series $\left\{x_{n}\right\}$, we construct a new sequence by mapping the original one through a binary alphabet. We symbolize with 1 a signal increase $\left(x_{n+1}>\right.$ $\left.x_{n}\right)$, and with 0 a decrease $\left(x_{n+1} \leq x_{n}\right)$. In case of ternary alphabet, 1 denotes the signal increase $\left(x_{n+1}>x_{n}\right), 0$ the decrease $\left(x_{n+1}<x_{n}\right)$ and 2 the signal invariance $\left(x_{n+1}=\right.$ $\left.x_{n}\right)$. To avoid the possible dependence of the encoded string on quantization procedure adopted to record the signal, a $p$ factor is introduced representing the minimum quantization level for a symbol change in the coded string. We considered the encoding parameter $p=0,0.005,0.01,0.02 \%$. The LZC index was computed 360 point-long FHR sequences (3 min).

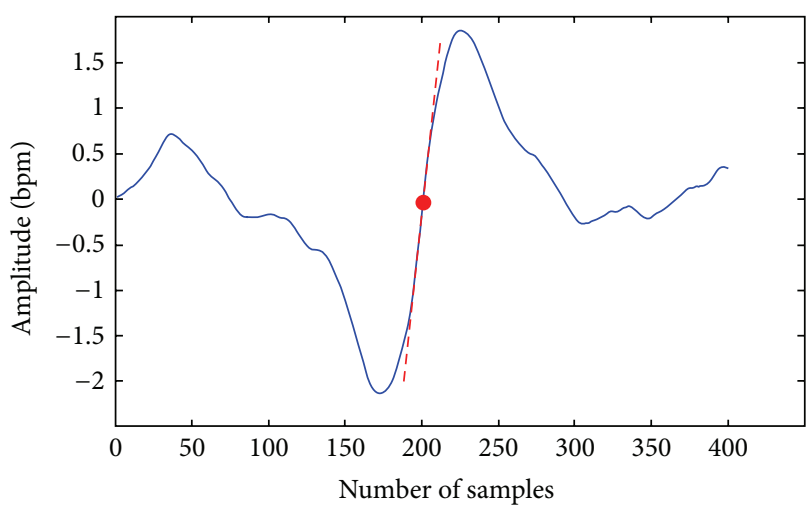

FIgURE 2: Phase Rectified Signal Average (PRSA) curve computed on a FHR recording. The computation of the Acceleration Phase Rectified Slope is shown: APRS is defined as the slope of the PRSA curve in the anchor point (red dot).

2.3.3. Phase Rectified Signal Average (PRSA). Phase rectified signal average (PRSA) is a technique introduced by Bauer et al. in 2006 [20]. It allows the detection and quantification of quasiperiodic oscillations in nonstationary signals affected by noise and artifacts, by synchronizing the phase of all periodic components. This method demonstrated its usefulness in FHR signal analysis, when episodes of increasing and/or decreasing FHR appear [21]. In fact, occurrence or absence of such periods can be related to the healthy status of the fetus. For this reason, we introduced the PRSA method to quantify fetal well-being states.

The PRSA curve is obtained from the HRV series. The procedure that can be followed to construct the curve is detailed and described in [20]. The great advantage given by the PRSA curve is the fact that a 30-40-minute HRV signal can be condensed in a single waveform, showing the average dynamic pattern of the recording under analysis. An example of PRSA curve is shown in Figure 2, where the red dot represents the anchor point and the dashed red line is the slope of the curve in the anchor point.

In order to construct the curve, we employed $200 \mathrm{sec}$ windows (total number of 400 samples) obtained from the FHR signal, which were selected if the right average of the window was bigger than the left average. Then, the windows were synchronized in their anchor point (the middle point of the curve) and averaged.

Starting from the PRSA curve, it is possible to compute several parameters that describe its shape and, indirectly, quantify the overall dynamics in the HRV series. Thus, those parameters can be employed to provide a clue about fetal behavior and well-being.

In [22], we proposed the Acceleration Phase Rectified Slope (APRS) and the Deceleration Phase Rectified Slope (DPRS), as useful indices computed on the PRSA curve in order to verify fetal well-being. For a detailed description of how these parameters are computed, please refer to [22].

Table 2 summarizes all the parameters we have considered in fetal HR analysis. Parameters have been grouped as Frequency domain (autoregressive power spectrum 
TABLE 2: Methods, extracted parameters, sequence lengths, and hypotheses for using the relevant parameter.

\begin{tabular}{|c|c|c|c|}
\hline Method & Parameters & Sequence length & Hypothesis \\
\hline $\begin{array}{l}\text { Frequency domain analysis: } \\
\text { periodogram and } \\
\text { autoregressive model } \\
\text { Measurement of spectral } \\
\text { components in defined } \\
\text { frequency bands }\end{array}$ & $\begin{array}{l}\% \text { of spectral power }\left(\mathrm{msec}^{2}\right) \text { in frequency bands: } \\
\text { Low frequency } 0.03-0.15 \mathrm{~Hz} \\
\text { Movement (activity) frequency } 0.15-0.5 \mathrm{~Hz} \\
\text { High frequency } 0.5-1 \mathrm{~Hz} \\
\mathrm{LF} /(\mathrm{MF}+\mathrm{HF})\end{array}$ & $\begin{array}{l}3 \text { min } \\
360 \text { values }\end{array}$ & $\begin{array}{l}\text { Quantification of the } \\
\text { activity of the autonomic } \\
\text { nervous system }\end{array}$ \\
\hline \multirow{2}{*}{$\begin{array}{l}\text { Time domain analysis: } \\
\text { morphological HR } \\
\text { modification and variability }\end{array}$} & $\begin{array}{l}\text { STV }(\mathrm{msec}) \\
\text { II }\end{array}$ & $\begin{array}{l}1 \mathrm{~min} \\
120 \text { values }\end{array}$ & $\begin{array}{l}\text { Variability in the short } \\
\text { period }\end{array}$ \\
\hline & $\begin{array}{l}\text { FHR avg (msec) } \\
\text { LTI (msec) }\end{array}$ & $\begin{array}{l}3 \text { min } \\
360 \text { values }\end{array}$ & $\begin{array}{l}\text { Variability in the long } \\
\text { period }\end{array}$ \\
\hline Approximate entropy & $\operatorname{ApEn}(m, r) m=1,2 ; r=0.1,0.15,0.2$ & $\begin{array}{c}3 \min \\
N=360 \text { values } \\
\end{array}$ & Recurrent patterns \\
\hline Sample entropy & $\operatorname{SampEn}(m, r) m=1,2 ; r=0.1,0.15,0.2$ & $\begin{aligned} & 3 \min \\
N= & 360 \text { values }\end{aligned}$ & $\begin{array}{l}\text { Recurrent patterns } \\
\text { Basis for investigating } \\
\text { repetitive patterns at } \\
\text { different time scales }\end{array}$ \\
\hline $\begin{array}{l}\text { Lempel Ziv complexity } \\
\text { (LZC) }\end{array}$ & $\begin{array}{l}\text { LZC binary or ternary coding } \\
\text { LZC }(2 \text { or } 3, P=0,0.005,0,01,0.2)\end{array}$ & Whole recording & $\begin{array}{c}\text { Rate of new patterns arising } \\
\text { with signal evolution in } \\
\text { time }\end{array}$ \\
\hline PRSA & Acceleration/Deceleration Phase Rectified Slope & Whole recording & Quasiperiodic oscillations \\
\hline
\end{tabular}

estimation-LF-power, MF-power, HF power, and LF/(MF $+\mathrm{HF})$ ); time domain (short term variability (STV), long term irregularity (LTI), Interval Index (II)); and regularity and complexity parameters (approximate entropy (ApEn), sample entropy (SampEn), Lempel Ziv complexity (LZC), and finally $P R S A$ parameters). All parameters are listed in Table 2 according to the time windows, which are suggested on the basis of our results.

For each group of them the pathophysiological meaning or the most reliable hypothesis is presented.

By this approach to the study of FHR we performed classification of different fetal states and we obtained diagnostic indications in pathologies such as intrauterine growth restriction (IUGR) and fetal distress [23, 24].

\section{Results}

Results are reported for the two groups of fetuses concerning the parameters illustrated in Sections: among the time parameters, STV, II, and LTI were selected; all frequency domains indices were computed by using the autoregressive power estimation (LF, MF, HF, and the ratio $\mathrm{LF} /(\mathrm{HF}+\mathrm{MF})$ ); among non-linear parameters, ApEn and SampEn were selected and compared to quantify non-linear complexity characteristics of FHR series; LZC parameters add information about complexity and predictability of FHR time series; finally, for the PRSA based parameters, APRS and DPRS were considered.

The target of the study was to identify which parameter or parameter set is most efficient in the discrimination between healthy and IUGR fetuses. Analysis of the FHR that consider more than one parameter at time has the objective to early identify signs of fetal distress that could bring interventions against possible life-threatening events.
In order to verify the ability of the selected parameters to discriminate between healthy and IUGR fetuses, we first verified that the two populations showed Gaussian distributions for all parameters using the Kolmogorov-Smirnov test, in order to further apply the $t$-test for the discrimination.

Table 3 summarizes the results concerning the healthy and IUGR groups of fetuses. Among the time parameters, both STV and LTI show great performance in the discrimination task (STV: $P$-value $=1.22 e-9 ;$ LTI: value $=1.5 e-11$ ), while Interval Index does not.

Results in frequency domain parameters show a weak capability to differentiate normal versus IUGR fetuses. Nevertheless, many results reported in the literature demonstrate their ability in assessing the cardiovascular well-being in adults. So they still remain important candidates to monitor cardiovascular regulation dynamics in FHR time series, although in this case they do not seem able to discriminate IUGR fetuses. As a matter of fact, the frequency parameters are related to physiological mechanisms acting on the heart control. So, measuring the HF component of the PSD is a way to measure respiratory fetal activity providing a parameter directly related to hypoxia or to a respiratory stress state.

The analysis of non-linear parameters shows that all considered parameters allow the rejection of the null hypothesis: $\operatorname{ApEn}(1,0.1)$ with $P$-value $5.14 e-07$, confirming to be highly sensitive to the IUGR condition, $\operatorname{LZC}(2,0)$ with $P$ value $7.8 e-4$, and $\operatorname{SampEn}(1,0.1)$ with $P$-value $2.08 e-7$, demonstrating a very high discriminant ability between the two groups.

Moreover, even similar analysis we did in a different population of normal and IUGR fetuses by using multiscale entropy approach [23] also provided satisfying levels of discrimination power of the entropy indices, thus confirming 
TABLE 3: Results of fetal HRV analysis by parameters in time domain, in frequency domain, by nonlinear indices and PRSA derived indices. Usefulness in separating populations is confirmed by $t$-test results.

\begin{tabular}{|c|c|c|c|c|}
\hline Parameter & $\begin{array}{c}\text { Healthy } \\
\text { (mean } \pm \text { std) }\end{array}$ & $\begin{array}{c}\text { IUGR } \\
(\text { mean } \pm \text { std })\end{array}$ & $t$-test & $P$ value \\
\hline \multicolumn{5}{|l|}{ Time parameters } \\
\hline $\mathrm{STV}(\mathrm{ms})$ & $6.7 \pm 2.24$ & $4.29 \pm 1.62$ & $* * *$ & $1.22 e-09$ \\
\hline Interval index & $0.87 \pm 0.07$ & $0.86 \pm 0.06$ & & 0.37 \\
\hline LTI (ms) & $21.46 \pm 6.53$ & $17.17 \pm 5.37$ & $* * *$ & $1.5 e-11$ \\
\hline \multicolumn{5}{|l|}{ Frequency domain } \\
\hline LF (Low Frequency power) & $82.92 \pm 5.29$ & $81.39 \pm 6.13$ & & 0.17 \\
\hline MF (Movement Frequency power) & $6.7 \pm 2.24$ & $11.61 \pm 3.50$ & & 0.63 \\
\hline HF (High Frequency power) & $5.45 \pm 3.18$ & $6.65 \pm 3.97$ & & 0.08 \\
\hline $\mathrm{LF} / \mathrm{HF}+\mathrm{MF}$ & $5.36 \pm 1.78$ & $4.89 \pm 1.76$ & & 0.16 \\
\hline \multicolumn{5}{|l|}{ Nonlinear parameters } \\
\hline $\operatorname{ApEn}(1,0.1)$ & $1.33 \pm 0.13$ & $1.21 \pm 0.11$ & $* *$ & $5.14 e-7$ \\
\hline Lempel Ziv complexity $(2,0)$ & $1.00 \pm 0.08$ & $0.94 \pm 0.09$ & $*$ & 0.00078 \\
\hline $\operatorname{SampEn}(1,0.1)$ & $1.3 \pm 0.19$ & $1.13 \pm 0.15$ & $* *$ & $2.08 e-7$ \\
\hline \multicolumn{5}{|l|}{ PRSA parameters } \\
\hline APRS & $0.17 \pm 0.041$ & $0.12 \pm 0.042$ & $* * *$ & $7.76 e-12$ \\
\hline DPRS & $-0.18 \pm 0.046$ & $-0.12 \pm 0.042$ & $* * *$ & $1.08 e-13$ \\
\hline
\end{tabular}

the diagnostic and clinical usefulness of this family of parameters.

Among PRSA parameters, both APRS and DPRS, were demonstrated to be highly selective for the separation of the two groups. The APRS allows the rejection of the null hypothesis with a $P$-value of $7.76 e-12$. The DPRS behaves even better, with a $P$-value of $1.08 e-13$. The DPRS is the parameter in the analyses which exhibits the smallest $P$-value in the discrimination between healthy and IUGR patients. On the contrary other PRSA parameters reported in the literature by Huhn et al. [21], when applied to our population of fetuses, are not efficient in the discrimination as already reported in [22].

Figure 3 shows the boxplots of the subset of parameters which show significant $P$-values $(P<0.05)$ computed in the analysis of the two groups of fetuses.

A further improvement of the diagnostic ability of our set of parameters could be obtained by a multivariate analysis, in which two or more parameters are considered together for the discrimination task. We did not perform a multiparametric analysis in depth for the many combinations of indices we computed, but we can support the previous claim by some preliminary results. Figure 4 shows as an example of what can be obtained by combining the discrimination power of two parameters: plot of $\operatorname{ApEn}(1,0.1)$ versus LTI values shows how healthy and IUGR populations can be separated, with very few errors, in different subspaces.

\section{The Future: Wearable Technology for Fetal Monitoring}

Monitoring fetal states can also be performed by measuring fetal ECG through electrodes placed over the maternal abdomen after the 26th week of pregnancy [25], which directly provide a measure of the FECG. Unfortunately, it is very difficult to reliably reveal this FECG both for the low SNR, due to noise superimposed and maternal ECG interference, and for the position of the fetus that almost continuously changes his position inside the uterus. The recording can be made only at the hospital and requires the presence of expert personnel. Even in that case, measurement of FECG remains a difficult task.

Nevertheless, recording the FECG could provide information on the beat structure (long QT, T wave morphology and slope), which is related to heart diseases and to hypoxic fetal states. Moreover, FECG recordings allow longer periods of HRV measurements with respect to CTG which employs ultrasounds (being the ECG completely noninvasive). The idea is to design a "Fetal Holter" for very long FHRV signal acquisitions.

With this focus, recent evolution in wearable technology has started to produce effects even in the biomedical devices field. As a matter of fact, these new wearable devices allow measuring several physiological parameters continuously in normal life conditions for long periods. Thus, interesting perspectives are now open toward the development of new systems, even in the field of fetal monitoring. With this focus, our research group has designed a new monitoring system, namely, the Telefetalcare system, that makes use of wearable technologies to measure FECG [26] through textile electrodes embedded in everyday garments.

A first example of what we can obtain by a wearable prenatal garment sensorized with 8 ECG textile electrodes and a miniaturized acquisition system is illustrated in Figure 5, where one lead of the fetal-maternal ECG is reported together with the QRS detection. Till now, the Telefetalcare has been used on a limited number of patients, showing good performances in both terms of quality of the acquired signals 


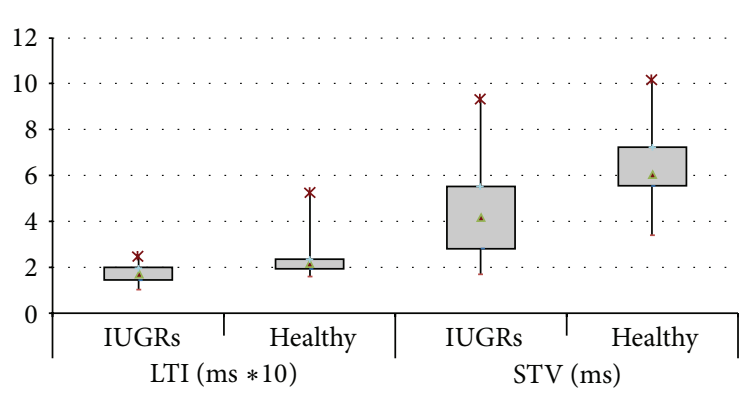

(a)

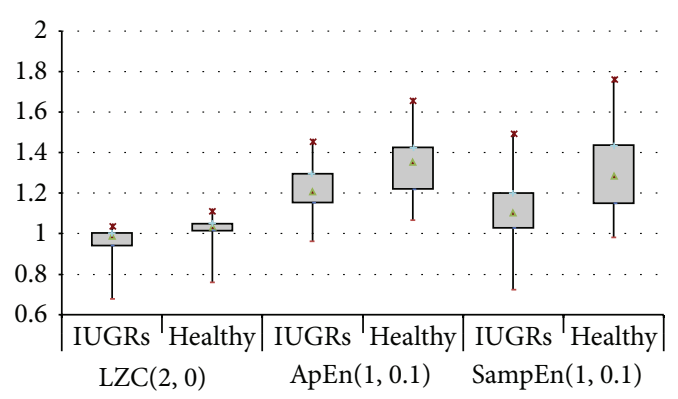

(b)

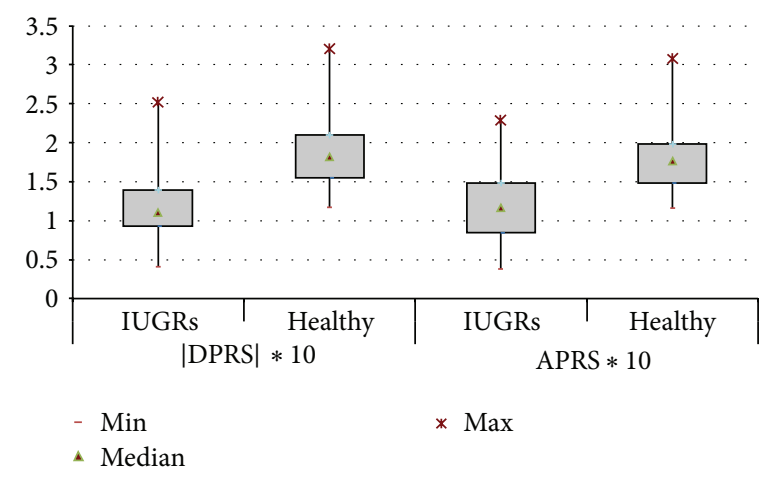

(c)

FIGURE 3: Boxplots of the significant parameters (the height of each box represents the distance between quartile $1(25 \%)$ and quartile $3(75 \%)$ ); the triangular marker is the median; $x$ denotes the maximum; and - marker is the minimum. (a) Diagram contains time domain indices, (b) diagram non linear indices and (c) diagram PRSA indices.

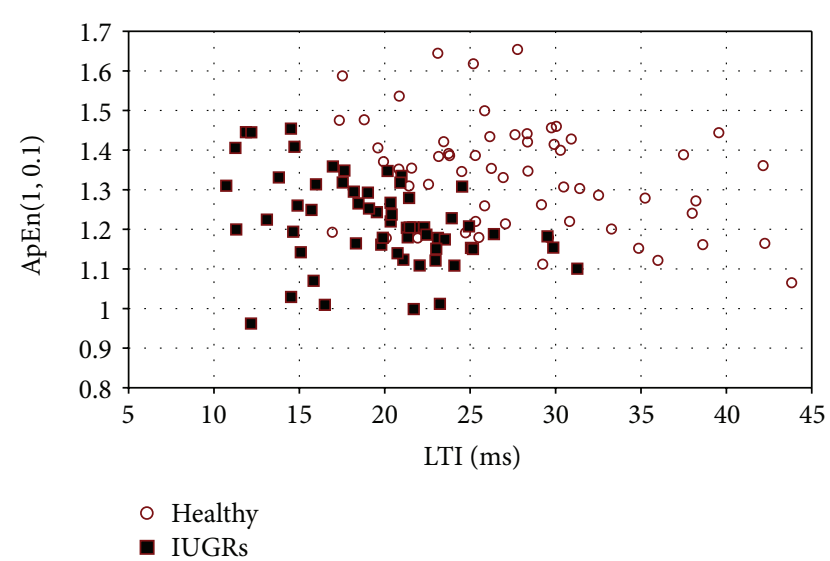

FIGURE 4: Individual data of ApEn(1,0.1) versus LTI. The two groups of IUGRs and healthy fetuses occupy different subspaces in the diagram and can be separated quite easily with very few errors.

and in terms of fetal QRSs detection. At the moment both the separation of fetal-maternal ECGs and the digital processing are performed offline on a notebook computer, using a graphical user interface implemented in Matlab environment.

The final goal of this novel approach is to produce a system that every pregnant woman can use at home, able to collect FECG signal, for long periods, in a comfortable way, and to send data to the hospital for evaluation, through a wireless link.

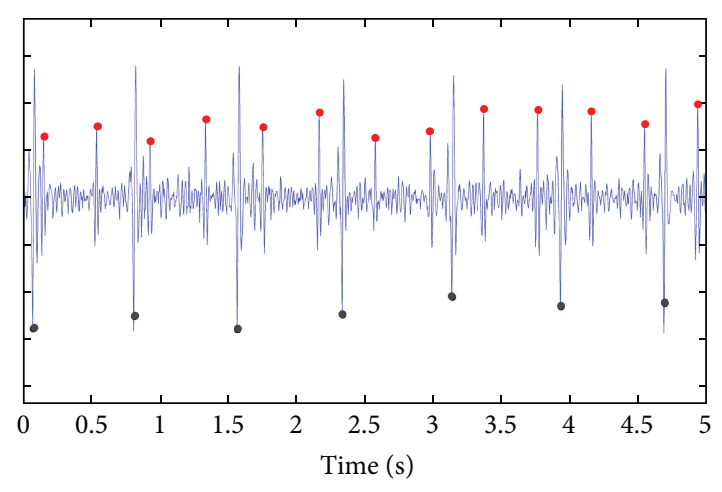

Figure 5: Example of ECG recording taken from the Telefetalcare system. The identification of maternal (gray dots, down) and fetal (red dots, up) heart beats is computed off-line by a novel algorithm implemented in Matlab.

Figure 6 illustrates the functional architecture of the whole system. Acquisition of the cardiac electric signals takes place through a dedicated hardware device which is wireless connected to the patient through the sensorized garment. To reduce the costs connected with the hardware manufacturing, the device has no display for user interface and only consists of an 8-channel differential amplifier, paired with a BluetoothTM wireless communication module. Smartphones or tablets available nowadays are endowed with high resolution color screens whose capabilities outpace 


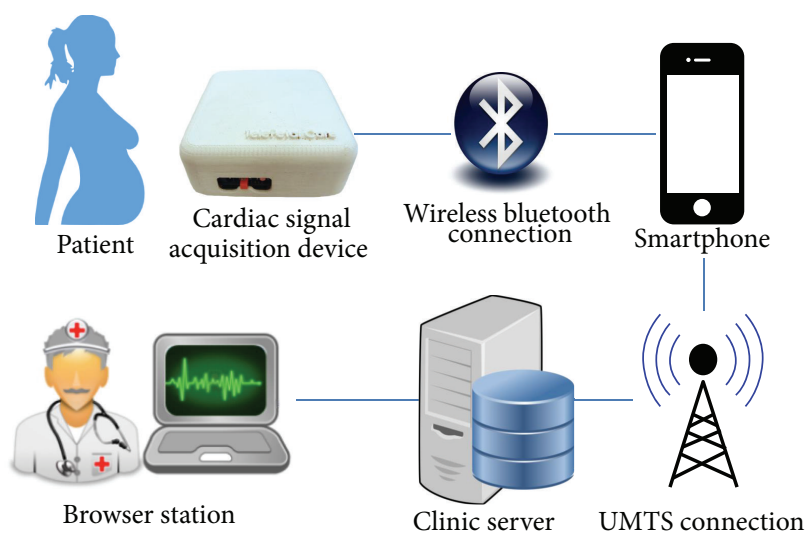

FIGURE 6: Actual architecture of the Telefetalcare system.

those of any other rendering device and computers available in the past decade.

Our objective is to obtain a high quality fetal ECG signal, for long periods, in an unsupervised environment (mother normal life) to extract fetal HRV in order to use it as an indicator of fetal well-being and/or stress conditions.

Of course, the analysis methods, previously presented and adopted for the fetal HRV signal from CTG recordings, will be used in the system postprocessing step. As a matter of fact, a significant improvement in the quality of fetal well-being assessment could be obtained by more frequent and accurate signal measurements and analysis, as costs in fetal monitoring will be drastically reduced.

\section{Discussion}

The paper presents results obtained from the application of several analysis tools to fetal heart rate variability signals. FHR signals were recorded through CTG in normal and IUGR fetuses, with the goal of demonstrating that fetal monitoring can be strongly improved by new analysis techniques and parameters related to pathophysiological fetal states.

The work evidenced some important points.

First, FHRV signal carries a lot of information about fetal condition during pregnancy and CTG, being the most employed technique supporting the diagnostic process along the final part of the pregnancy, and allows extracting this information through an accurate analysis. We considered a population including 61 normal and 61 IUGR subjects and we checked different approaches to find out reliable indices for separating the two groups. We tested time domain, frequency domain, and nonlinear approaches and results showed that time domain and nonlinear indices significantly separate the two groups allowing a clear classification. This is very important as early identification of IUGR condition allows proper intervention reducing life-threatening events.

However, not all parameters are equally sensitive to evolving fetal conditions. Entropy parameters, Lempel Ziv complexity indices, variability parameters in time domain, and PRSA derived indices exhibit excellent performance in classification of normal and IUGR population. Nevertheless it is necessary to stress the importance of considering a quite large set of parameters to investigate the complex regulation of the fetal cardiovascular system. The interaction with the placenta, thus with the mother circulation, and the development of the controlling systems in the fetus are all factors influencing and acting on the fetal state.

Results and examples shown in the paper clearly suggest that monitoring systems could be improved by adding diagnostic and classification power through advanced signal processing techniques.

In particular, we want to stress the importance of adopting a multiparameter analysis to better identify fetal states for the sake of preventing disease insurgence. Our preliminary analysis (ApEn/LTI in Figure 4) shows how the simple combination of two parameters can improves the identification of IUGR subjects from healthy ones. These aspects deserve future investigations through a multivariate analysis.

Another important point relies on the general use the proposed approach could have in the fetal HR analysis as CTG data are routinely measured during pregnancy. As a matter of fact, analysis tools can complement the clinical routine steps, providing further indications to physicians and nurses.

Our experience has shown that implementing advanced signal processing techniques can provide better classification results of the fetal states either in a normal development of the pregnancy (activity-quiet) [13] (vibroacoustic stimulation) [23] or in pathological conditions (distressed fetuses) [24] (IUGRs) [27, 28].

Moreover, the intrinsic complexity which characterizes fetal life and the possible associated diseases complicates the prediction and control of fetal development. To face this problem we need to develop more personalized monitoring system allowing an almost continuous noninvasive evaluation of the fetal state and in which knowledge based systems contribute to the care improvement.

As a further contribution to a knowledge based fetal monitoring approach, supported by an advanced technology, we have briefly presented a fetal ECG monitoring system, Telefetalcare, based on wearable technology and designed to permit an accurate and continuing assessment of fetal wellbeing. Advantages are in the signal quality with the direct measurement of fetal HRV and the long-term monitoring that can be easily performed. A wearable garment equipped with textile electrodes will allow pregnant women to monitor fetus health state without moving to the hospital, always having the clinician remote support.

The system can contribute to reducing costs of fetal monitoring still maintaining a significant quality or even improving the fetal wellbeing assessment.

These novel approaches can open a new window on the continuous monitoring of fetal development: further information can be extracted by introducing novel analysis tools, more sensitive to fetal states both in healthy and stress conditions, by increasing length, frequency, and quality of monitoring session. Methods and technological advancements both have a key role contributing to reaching this important scientific and social objective. 


\section{Conflict of Interests}

The authors declare that there is no conflict of interests regarding the publication of this paper.

\section{References}

[1] Task Force of the European Society of Cardiology and the North American Society of Pacing and Electrophysiology, "Heart rate variability: standards of measurement, physiological interpretation, and clinical use," Circulation, vol. 93, no. 5, pp. 1043-1065, 1996.

[2] G. Baselli, A. Porta, and S. Cerutti, "Spectral decomposition in multichannel recordings based on multivariate parametric identification," IEEE Transactions on Biomedical Engineering, vol. 44, no. 11, pp. 1092-1101, 1997.

[3] H. Kantz, J. Kurths, and G. Mayer-Kress, Eds., Nonlinear Analysis of Physiological Data, Springer, Berlin, Germany, 1998.

[4] D. Kaplan and L. Glass, Understanding Nonlinear Dynamics, Springer, New York, NY, USA, 1995.

[5] T. H. Mäkikallio, T. Seppänen, K. E. Airaksinen et al., "Dynamic analysis of heart rate may predict subsequent ventricular tachycardia after myocardial infarction," American Journal of Cardiology, vol. 80, no. 6, pp. 779-783, 1997.

[6] G. S. Dawes, C. R. S. Houghton, and C. W. G. Redman, "Baseline in human fetal heart-rate records," British Journal of Obstetrics and Gynaecology, vol. 89, no. 4, pp. 270-275, 1982.

[7] R. Mantel, H. P. Van Geijn, F. J. M. Caron, J. M. Swartjes, E. E. Van Woerden, and H. W. Jongsma, "Computer analysis of antepartum fetal heart rate: 1. Baseline determination," International Journal of Bio-Medical Computing, vol. 25, no. 4, pp. 261-272, 1990.

[8] R. Mantel, H. P. Van Geijn, F. J. M. Caron, J. M. Swartjes, E. E. Van Woerden, and H. W. Jongsma, "Computer analysis of antepartum fetal heart rate: 2. Detection of accelerations and decelerations," International Journal of Bio-Medical Computing, vol. 25, no. 4, pp. 273-286, 1990.

[9] D. Arduini, G. Rizzo, G. Piana, A. Bonalumi, P. Brambilla, and C. Romanini, "Computerized analysis of fetal heart rate: I. description of the system (2CTG)," Matern. Fetal.Invest, vol. 3, article 159, 1993.

[10] J. De Haan, J. H. Van Bemmel, B. Versteeg et al., "Quantitative evaluation of fetal heart rate patterns. I. Processing methods," European Journal of Obstetrics and Gynecology and Reproductive Biology, vol. 1, no. 3, pp. 95-102, 1971.

[11] K. J. Dalton, G. S. Dawes, and J. E. Patrick, "Diurnal, respiratory, and other rhythms of fetal heart rate in lambs," American Journal of Obstetrics and Gynecology, vol. 127, no. 4, pp. 414-424, 1977.

[12] S. Y. Yeh, A. Forsythe, and E. H. Hon, "Quantification of fetal heart beat-to-beat interval differences," Obstetrics and Gynecology, vol. 41, no. 3, pp. 355-363, 1973.

[13] M. G. Signorini, G. Magenes, S. Cerutti, and D. Arduini, "Linear and nonlinear parameters for the analysis of fetal heart rate signal from cardiotocographic recordings," IEEE Transactions on Biomedical Engineering, vol. 50, no. 3, pp. 365-374, 2003.

[14] J. M. Hausdorff and C.-K. Peng, "Multiscaled randomness: a possible source of $1 / \mathrm{f}$ noise in biology," Physical Review E, vol. 54, no. 2, pp. 2154-2157, 1996.

[15] J. Beran, Statistics for Long-Memory Processes, Chapman v Hall, New York, NY, USA, 1994.
[16] S. M. Pincus, "Approximate entropy (ApEn) as a complexity measure," Chaos, vol. 5, no. 1, pp. 110-117, 1995.

[17] J. S. Richman and J. R. Moorman, "Physiological time-series analysis using approximate and sample entropy," American Journal of Physiology: Heart and Circulatory Physiology, vol. 278, no. 6, pp. H2039-H2049, 2000.

[18] M. Costa, A. L. Goldberger, and C.-K. Peng, "Multiscale entropy analysis of complex physiologic time series"', Physical Review Letters, vol. 89, no. 6, Article ID 068102, 2002.

[19] A. Lempel and J. Ziv, "Multiscale entropy analysis of complex physiologic time series," IEEE Transactions on Information Theory, vol. 22, no. 1, pp. 75-81, 1976.

[20] A. Bauer, J. W. Kantelhardt, A. Bunde et al., "Phase-rectified signal averaging detects quasi-periodicities in non-stationary data," Physica A, vol. 364, pp. 423-434, 2006.

[21] E. A. Huhn, S. Lobmaier, T. Fischer et al., "New computerized fetal heart rate analysis for surveillance of intrauterine growth restriction," Prenatal Diagnosis, vol. 31, no. 5, pp. 509-514, 2011.

[22] A. Fanelli, G. Magenes, M. Campanile, and M. G. Signorini, "Quantitative assessment of fetal well-being through CTG recordings: a new parameter based on Phase Rectified Signal Average," IEEE Journal of Biomedical and Health Informatics, vol. 17, no. 5, pp. 959-966, 2013.

[23] G. Magenes, M. G. Signorini, D. Arduini, and S. Cerutti, "Fetal heart rate variability due to vibroacoustic stimulation: linear and nonlinear contribution," Methods of Information in Medicine, vol. 43, no. 1, pp. 47-51, 2004.

[24] M. Ferrario, M. G. Signorini, G. Magenes, and S. Cerutti, “Comparison of entropy-based regularity estimators: application to the fetal heart rate signal for the identification of fetal distress," IEEE Transactions on Biomedical Engineering, vol. 53, no. 1, pp. 119-125, 2006.

[25] S. Cerutti, G. Baselli, and S. Civardi, "Variability analysis of fetal heart rate signals as obtained from abdominal electrocardiographic recordings," Journal of Perinatal Medicine, vol. 14, no. 6, pp. 445-452, 1981.

[26] A. Fanelli, M. Ferrario, L. Piccini et al., "Prototype of a wearable system for remote fetal monitoring during pregnancy," in Proceedings of the 32nd Annual International Conference of the IEEE Engineering in Medicine and Biology Society (EMBC '10), pp. 5815-5818, September 2010.

[27] M. Ferrario, M. G. Signorini, and G. Magenes, "Comparison between fetal heart rate standard parameters and complexity indexes for the identification of severe intrauterine growth restriction," Methods of Information in Medicine, vol. 46, no. 2, pp. 186-190, 2007.

[28] M. Ferrario, M. G. Signorini, and G. Magenes, "Complexity analysis of the fetal heart rate variability: early identification of severe intrauterine growth-restricted fetuses," Medical and Biological Engineering and Computing, vol. 47, no. 9, pp. 911-919, 2009. 


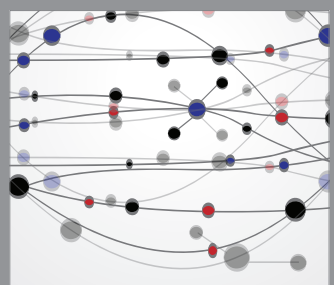

The Scientific World Journal
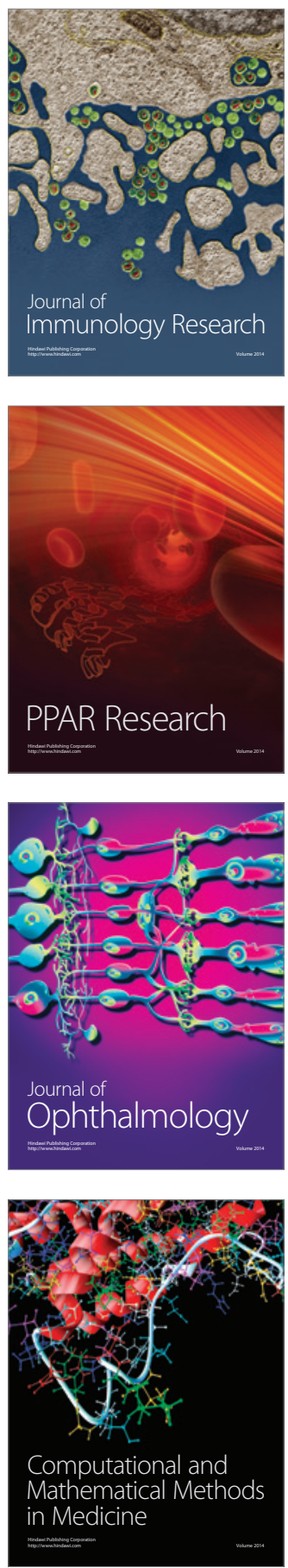

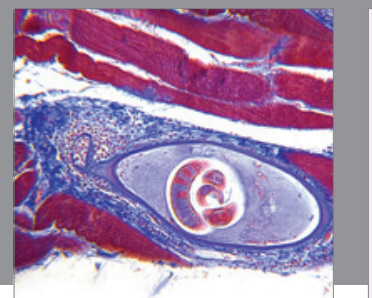

Gastroenterology

Research and Practice
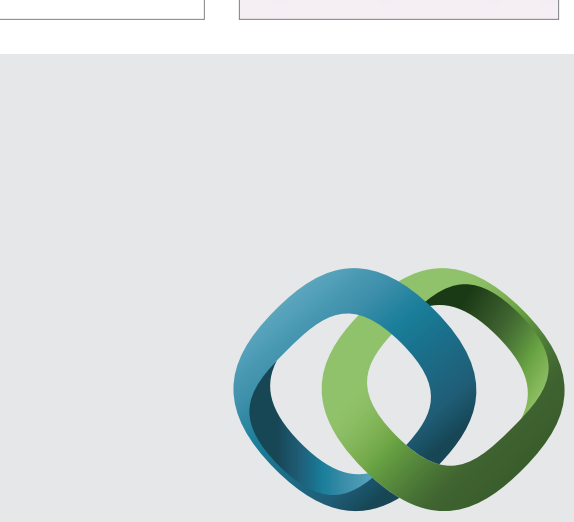

\section{Hindawi}

Submit your manuscripts at

http://www.hindawi.com
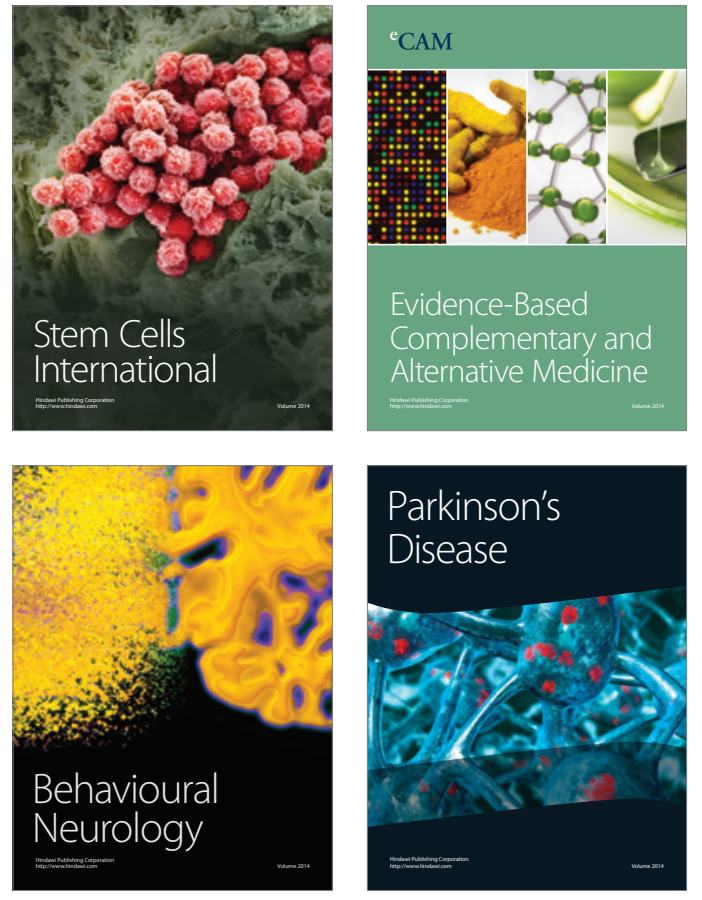
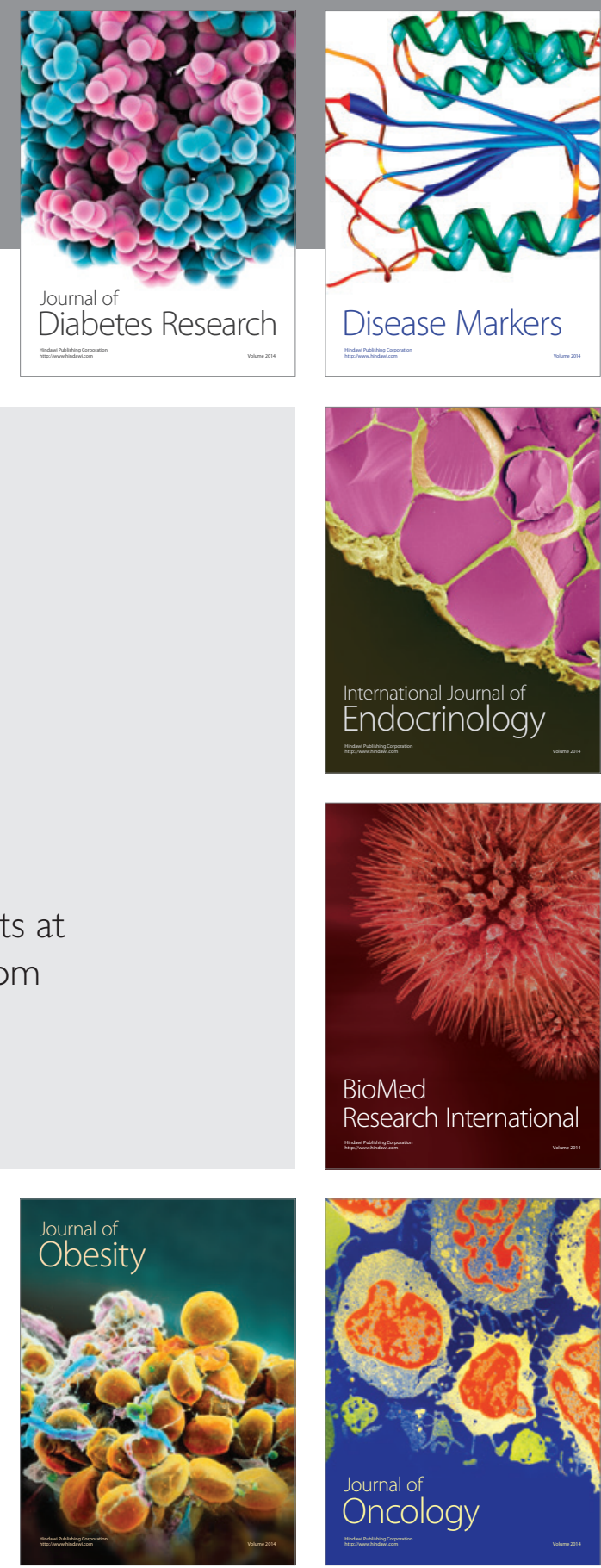

Disease Markers
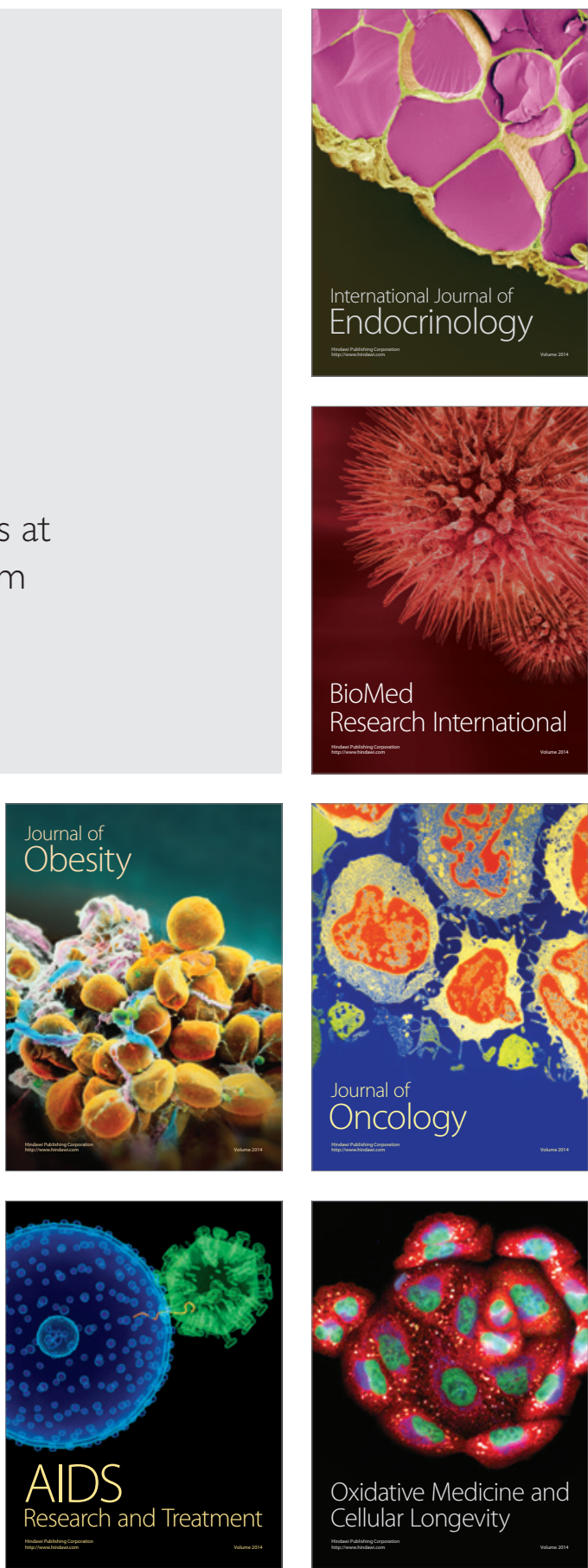